\title{
Photoprotection and triplet energy transfer in higher plants: the role of electronic and nuclear fluctuations
}

\author{
Lorenzo Cupellini*, Sandro Jurinovich, Ingrid G. Prandi, Stefano Caprasecca and Benedetta Mennucci \\ Dipartimento di Chimica e Chimica Industriale, University of Pisa \\ *Iorenzo.cupellini@for.unipi.it
}

\begin{abstract}
The quenching of Chlorophyll triplets by triplet energy transfer (TET) to carotenoids is one of the photoprotection strategies in photosynthetic organisms, and prevents singlet oxygen formation.

Here we present the study of TET rates in a minor lightharvesting complex (LHC) of higher plants, using a fully atom- istic strategy that combines a molecular dynamic simulation a polarizable quantum/classical calculation.

We find that structural fluctuations of the LHC can largely enhance the TET rates, which are in the sub-nanosecond scale, in agreement with experimental findings.
\end{abstract}

Photosynthetic organisms employ several photoprotection strategies to avoid damage due to the excess energy in high light conditions. Among these, quenching of triplet clorophylls (Chls) by neighboring carotenoids (Cars) is fundamental in preventing the formation of singlet oxygen.

Singlet excited $\mathrm{Chl} *$ can decay into triplets (3 Chl*) which sensitize molecular oxygen to form singlet oxygen, which induces damage in its local environment by destroying lipids and nucleic acids and proteins.[1], [2], [3] Cars are able to accept the triplets from chlorophylls (chls) by triplet energy transfer (TET), and dissipate the excess energy to heat.[4] The efficiency of Chl triplet quenching is $95 \%$ in antenna complexes of Photosystem II in higher plants, and the timescale of TET from Chls to Cars has been found to be faster than 500 ps in the major light-harvesting complex of Photosystem II (LHCII).[5]

TET is a spin-allowed process that consists in the transfer of a triplet configuration from a donor to an acceptor molecule. Because TET is based on the Dexter-like mechanism of electron exchange, and it requires an overlap between the molecular orbitals of donor and acceptor, thermal fluctuations are expected to play a relevant role in determining the coupling distribution. Here, we present a fully atomistic strategy, combining classical molecular dynamics (MD) with a hybrid time-dependent density functional theory (TDDFT)/polarizable MM description, to describe TET in the natural environment of the LHC.

In particular, we focused on CP29 (or Lhcb4), a minor lightharvesting complex of the Photosystem II whose crys- tal structure was recently obtained by Pan et al. at high resolution. [6] CP29 contains two strongly couplled Car- Chl clusters, namely those formed by Lutein (Lut) and Violaxanthin (Vio) with the three closest Chls. These two clusters are characterized by a similar arrangement of the Chls around the Car (See Figure 3).
The rate of the TET process can be related to the electronic triplet coupling by Fermi's Golden Rule:

$$
\begin{array}{lll}
\text { kTET } & 2 \pi & J D A \\
= & \frac{2}{n|V D A|}
\end{array}
$$

Where VDA is the electronic coupling between initial and final states and JDA is the spectral overlap between the Franck-Condon weighted densities of states of donor and acceptor. Here we employ the fragment spin difference (FSD) scheme, a method to compute accurate triplet couplings starting from the eigenstates of the electronic Hamiltonian, namely the adiabatic states. [7] The spectral overlap was obtained from spectroscopic data.

We computed the TET couplings along 100 uncorrelated frames of an $80 \mathrm{~ns}$ MD simulation. In Figure 1 we compare the MD rms (/ ( $\overline{2}$ )) couplings with those computed on the crystal structure. In all pairs, except Lut-Chl a610, the MD average coupling is larger than the corresponding one obtained from the crystal. In particular, the coupling between Vio and Chl a603 nearly shows a six-fold increase. This is due to a limited number of favorable configurations with very large coupling values: in fact, excluding the largest

10 couplings result in a $40 \%$ drop of the rms coupling, indicating that these configurations account for more than half of the average TET rate.

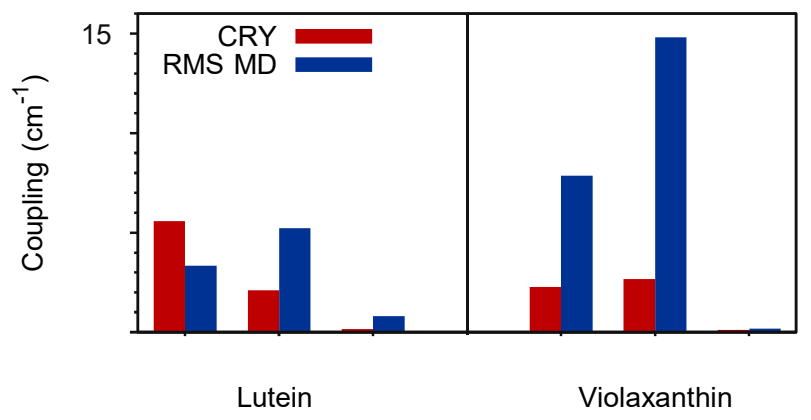

Fig. 1. Comparison between the crystal structure couplings (red) and theMD averaged couplings (blue).

A geometrical analysis of the TET can be performed using the volume of the intersection between the Van der Waals regions of the interacting pigments, defined as a union of interlocking spheres positioned on the atoms of 


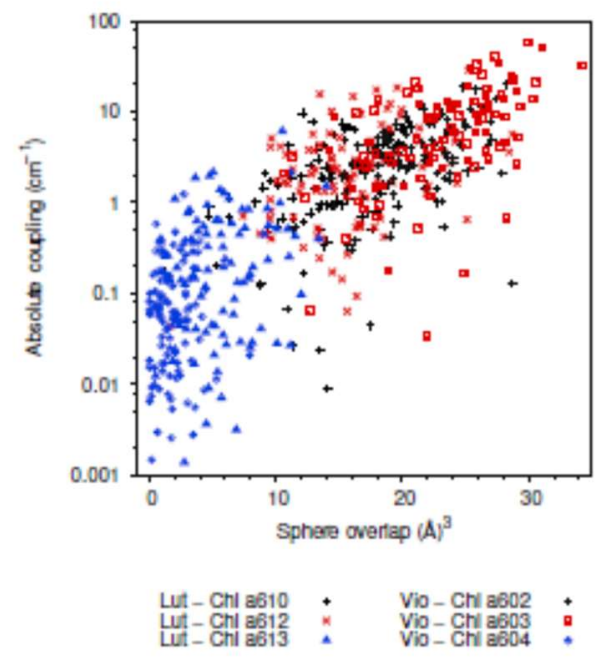

Fig. 2. Scatter plot of absolute coupling values (logarithmic scale) versus geometric overlap. Different Car-Chl pairs are shown in different colours.

the $\pi$-backbone with a radius 1.4 times the Van der Waals radius of the atom. To see if the geometric overlap can explain the coupling fluctuations, in Figure 2 we correlate the absolute coupling of all Car-Chl pairs to the geometric overlap. Despite the simplicity of this model, the magnitude of the coupling generally follows the geometric overlap.

The coupling values presented and discussed above are here used to compute the TET rates as obtained from (1), where $\mathrm{V}_{B A}$ is an average of the squared couplings along the MD. All the results are reported in Table I. In the same Table we also report the TET times obtained from the couplings calculated at the crystal structure. Figure 3 shows the transfer times and relates them to the arrangement of the pigments. In all pairs, except Lut-Chl a610 and a603-a609, the MD average time is shorter than the corresponding one obtained from the crystal structure, by more than one order of magnitude.

These data correlate well with experimental observations. The timescale of TET from Chls to Cars have in fact been found to be faster than $500 \mathrm{ps}$ in the major light harvesting complex of Photosystem II (LHCII).[5] Our results show that the TET quenching mechanism strongly depends on the fluctuations of the surrounding environment. Notably, relying on the crystal structures may result in an underestimate of TET couplings and rates.
TABLE I

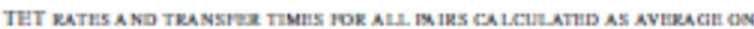

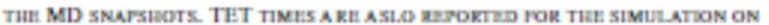
THE CKYSTAL STRUCTURE (TIMERCOY)

\begin{tabular}{|c|c|c|c|c|}
\hline \multicolumn{2}{|c|}{ Pair } & $k \operatorname{rin}\left(s^{-1}\right)$ & time a MD & time $\Leftrightarrow \mathrm{Cr} y$ \\
\hline \multirow{3}{*}{ Lat } & a 610 & $2.13 \times 10^{\rho}$ & $470 \mathrm{ps}$ & $170 \mathrm{ps}$ \\
\hline & $\mathrm{a} 612$ & 5. $24 \times 10^{\rho}$ & $190 \mathrm{ps}$ & $1.2 \mathrm{~ns}$ \\
\hline & af 613 & $1.21 \times 10^{8}$ & $8.2 \mathrm{~ns}$ & $260 \mathrm{~ns}$ \\
\hline \multirow{3}{*}{ Vio } & $\mathrm{a} 602$ & $1.14 \times 10^{10}$ & $140 \mathrm{ps}$ & $1.1 \mathrm{~ns}$ \\
\hline & ato03 & $4.05 \times 10^{10}$ & $25 \mathrm{ps}$ & $760 \mathrm{ps}$ \\
\hline & afol 4 & 5. $20 \times 10^{A}$ & $190 \mathrm{~ns}$ & $540 \mathrm{~ns}$ \\
\hline
\end{tabular}

Fig. 3. TET time constants for the pairs investigated in this work. The thickness of the lines connecting the pigments represents the order of magnitude of the transfer time constant. 


\section{ACKNOWLEDGMENT}

This work has been accepted for publishing [8]. L.C., S.J., S.C. and B.M. acknowledge the European Research Council (ERC) for financial support in the framework of the Starting Grant (EnLight - 277755). I.G.P. acknowledges CNPq Brazil for PhD scholarship (236693/2012-3).

\section{REFERENCES}

[1] M. Ballottari, M. Mozzo, J. Girardon, R. Hienerwadel, and R. Bassi, "Chlorophyll triplet quenching and photoprotection in the higher plant monomeric antenna protein Lhcb5.," J. Phys. Chem. B, vol. 117, pp. 11337-48, Sept. 2013.

[2] R. Croce and H. van Amerongen, "Natural strategies for photosynthetic light harvesting.," Nat. Chem. Biol., vol. 10, pp. 492501, June 2014.

[3] M. Mozzo, L. Dall'Osto, R. Hienerwadel, R. Bassi, and R. Croce, "Photoprotection in the antenna complexes of photosystem II: role of individual xanthophylls in chlorophyll triplet quenching.," J. Biol. Chem., vol. 283, pp. 6184-92, Mar. 2008.

[4] R. Bittl, E. Schlodder, I. Geisenheimer, W. Lubitz, and R. J. Cogdell, "Transient EPR and Absorption Studies of Carotenoid Triplet Formation in Purple Bacterial Antenna Complexes," J. Phys. Chem. B, vol. 105, pp. 5525-5535, June 2001.

[5] R. Scho" del, K. D. Irrgang, J. Voigt, and G. Renger, "Rate of carotenoid triplet formation in solubilized light-harvesting complex II (LHCII) from spinach.," Biophys. J., vol. 75, pp. 3143-53, Dec. 1998.

[6] X. Pan, M. Li, T. Wan, L. Wang, C. Jia, Z. Hou, X. Zhao, J. Zhang, and W. Chang, "Structural insights into energy regulation of lightharvesting complex CP29 from spinach.," Nat. Struct. Mol. Biol., vol. 18 , pp. 309-

15, Mar. 2011

[7] Z.-Q. You and C.-P. Hsu, "The fragment spin difference scheme for triplet-triplet energy transfer coupling.," J. Chem. Phys., vol. 133, p. 074105, Aug. 2010

[8] L. Cupellini, S. Jurinovich, I. G. Prandi, S. Caprasecca, and B. Mennucci, "Photoprotection and triplet energy transfer in higher plants: the role 